
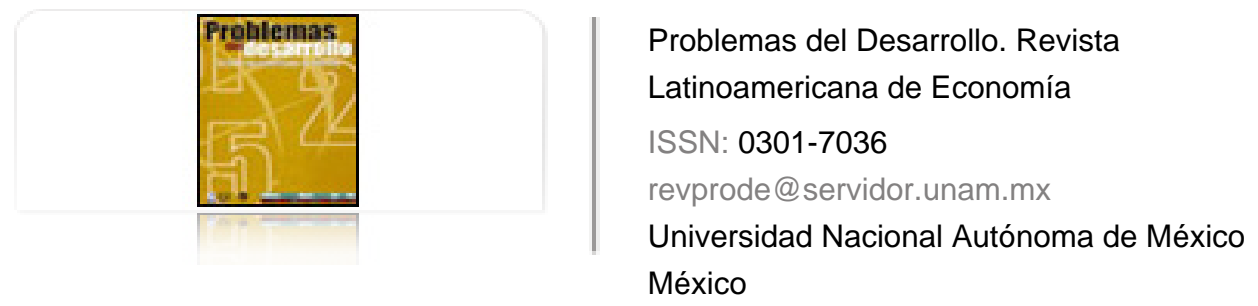

Saludjian, Alexis

Del MERCOSUR al ALCA: críticas al modelo liberal de nuevo regionalismo del BID Problemas del Desarrollo. Revista Latinoamericana de Economía, vol. 36, núm. 141, abril-junio, 2005, pp. 9-25

Universidad Nacional Autónoma de México

Distrito Federal, México

Disponible en: http://www.redalyc.org/articulo.oa?id=11820075002

Cómo citar el artículo

- Número completo

- Más información del artículo

Página de la revista en redalyc.org

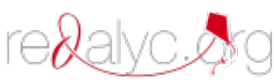

Sistema de Información Científica

Red de Revistas Científicas de América Latina, el Caribe, España y Portugal Proyecto académico sin fines de lucro, desarrollado bajo la iniciativa de acceso abierto 


\title{
Del MERCOSUR AL ALCA: CRÍTICAS AL MODELO LIBERAL DE
} NUEVO REGIONALISMO DEL BID

\author{
Alexis Saludjian*
}

Fecha de recepción: 27 de marzo de 2004. Fecha de aceptación: 24 de mayo de 2005.

\section{Resumen}

En la primera sección se discute un modelo del Banco Interamericano de Desarrollo (BID) en favor del ALCA, sobre la base de la aplicación de la teoría de economía geográfica al proceso de integración económica del Cono Sur de América Latina (MERCOSUR). En la segunda sección, abordamos los límites de este modelo, insistiendo en la ausencia de ciertas variables fundamentales para el estudio de las economías de estas regiones. Por cierto, los aspectos de las economías industrial internacional, del desarrollo y de la inserción de estos países de la periferia en la economía mundial, son esenciales para analizar el régimen de acumulación dominado por el espacio financiero que la mayoría de las naciones latinoamericanas experimentan, así como el proceso de integración de América.

Palabras clave: modelos de economía geográfica, MERCOSUR, régimen de acumulación a dominante financiera, $A L C A$.

\section{Résumé}

Nous discutons, dans la première section, un modèle de la Banque Inter-Américaine de Développement (BID) qui souligne les avantages de l'ALCA à partir de l'application de l'approche de l'économie géographique au processus d'intégration économique du Cône Sud d'Amérique Latine (le MERCOSUR). Nous soulignons, dans la seconde section, l'absence de variables fondamentales à l'étude des économies de cette région dans ce modèle. En effet, les aspects d'économie industrielle internationale, d'économie du développement, de la spécificité de l'insertion dans l'économie mondiale des pays financiarisés de la périphérie sont essentiels à l'analyse du régime d'accumulation à dominante financière qui régit les économies de la plupart des pays latino-américains et l'intégration en cours dans les Amériques.

Mots clés: modèle d'économie géographique, MERCOSUR, régime d'accumulation à dominante financière, $A L C A$.

* Doctor en Ciencias Económicas, Universidad de París, 13 Villetaneuse, UfR Sciences Economiques et Gestion CEPN CNRS UMR 7115 ET GREITD. Correos electrónicos: saludjian@seg.univ paris13.fr y alex_saludj@yahoo.fr 


\begin{abstract}
On the basis of applying the economic geography approach to MERCOSUR's economic integration process, in the first section I discuss a model developed by the Inter-American Development Bank (IADB) in favor of $A L C A$. In the second section, we consider the limits of such a conception, noting the absence of certain fundamental variables that are nevertheless worthy of consideration in a study of the economies of the region. Indeed, the international industrial economic aspects, development economics, and the insertion into the world economy of these peripheral countries are of great importance in the finance-led accumulation regime that most Latin America countries experience and in the integration process in the Americas.
\end{abstract}

Key words: economic geography model, MERCOSUR, finance-led accumulation regime, ALCA.

\title{
Resumo
}

Discute-se um modelo do Banco Interamericano de Desenvolvimento (BID) em favor do ALCA, sobre a base da aplicação da teoria de economia geográfica ao processo de integração econômica do Cono Sul da América Latina (MERCOSUL) e abordam-se os limites de este modelo insistendo na ausência de certas variáveis fundamentais para o estudo das economias dessas regiões. Atenção, os aspectos das economias industrial internacional, do desenvolvimento e da ineserção de estes paises da periferia na economia mundial são vitais para analizar o regime de acumulação dominado pela finança que a maioria das nações da América Latina sofrem e o processo de integração da América.

Palavras chave: modelos de economia geográfica, MERCOSUL, regime de acumulação a dominante financeira, ALCA. 


\section{Introducción}

$\mathrm{E}^{\prime}$

1 Área de Libre Comercio de las Américas (ALCA) tenía que ser implantada en acuerdos que institucionalizarían el ALCA todavía están lejos de ser firmados. Sin embargo, el prinicipal interesado, Estados Unidos, espera, al menos, un ALCA light, como demostró su secretaria de Estado en la visita que realizó a los principales países de América del Sur en abril de 2005.

Así, el MERCosur, creado en 1991 por el Tratado de Asunción entre Argentina, Brasil, Paraguay y Uruguay, tendría que disolverse en el ALCA o, por lo menos, abrir sus mercados de bienes y servicios a las demás naciones y, particularmente, a Estados Unidos. Considerando el dinamismo del acuerdo comercial del Cono Sur, medido con la tasa de comercio intramERCOSUR sobre el total del comercio, las cifras en 2002 (11.4\%) vuelven por debajo del nivel de 1991, antes de dicho tratado (13.1\%). Con base en estos problemas que enfrenta el MERCOSUR como bloque regional, este discurso y los modelos a favor del ALCA siguen teniendo fuerza.

Al final de la década perdida, el contexto económico en el Cono Sur determinará las características de su integración regional. El tipo de regionalización que tuvo lugar en el MERCOSUR estuvo profundamente marcado por el recurso casi exclusivo a los mercados, lo cual subrayó la dificultad de tratar el concepto regionalismo abierto. Ciertos economistas ven allí un oxímoron, de tan opuestos ${ }^{2}$ que parecen los términos que lo componen.

El nuevo regionalismo encuentra sus fundamentos en la nueva economía geográfica y privilegia el papel de la apertura y del progreso tecnológico para discutir acerca de la localización de las actividades en el espacio considerado.

El concepto regionalismo abierto, desarrollado por los neoestructuralistas de la CEPAL (1994), trata la integración económica latinoamericana con base en temas caros a los años 1950 (complementariedad productiva, políticas de coordinación macroeconómica, cierto grado de protección aduanera frente a terceros países), pero se inspira también en las teorías del nuevo regionalismo (apertura económica).

El objetivo de este artículo es presentar el tipo de regionalismo que prevaleció en el Cono Sur y subrayar las tensiones entre regionalismo y apertura económica de las nacio-

1 Durante el proceso de dictamen, el autor recibió algunas indicaciones de parte de sus árbitros y puntualmente actualizó ciertos datos.

2 Tal es el caso del economista T. Srinivaran, citado en Kuwayama (1999:14).

\section{Desaarrollo}


nes del MERCosur. En la primera sección se analiza la aplicación de un modelo de nuevo regionalismo (desarrollado por el BID) al MERCosur. En la segunda, evaluaremos los aportes y límites de dicho modelo. Así, veremos que la propuesta del BID insiste únicamente en los efectos teóricamente positivos de la apertura, sin tomar en consideración ideas estructuralistas de enfoque heterodoxo referentes a la red de seguridad regional en caso de crisis. En las conclusiones, presentaremos argumentos para criticar, en el contexto de la presión para la firma del ALCA, el modelo desarrollado por el BID, que legitima la estrategia estadounidense para las Américas.

\section{La integración regional del MERCOSUR según el BID en la perspectiva del ALCA}

El modelo del BID (desarrollado por Estevadeoral et al., 2000) se inscribe en la visión de un nuevo regionalismo definido por Ethier (1998) y se basa en la nueva teoría del comercio internacional (Krugman, 1991c y 1995). Los intentos de aplicación de la economía por medio de los modelos gravitacionales ${ }^{3}$ arrojan resultados más que insatisfactorios. De este modo, Darrigues y Montaud (2001) previeron, sobre la base de este modelo, una reubicación en 1999 de las actividades industriales hacia Argentina en detrimento de Brasil en los años 2000-2002. Actualmente, las fuerzas endógenas (centrípetas o centrífugas) se muestran muy desorientadas por las especificidades de América Latina. De la misma manera, Terra y Gigliotti (1995), que no consiguen conclusiones relevantes, afirman: "este primer esfuerzo, con la intención de ajustar un modelo para discutir problemas de localización geográfica de la producción provocados por la desaparición de barreras entre países, al final es extremadamente imperfecto aún si se aportan algunos elementos de reflexión" (p. 103). Los trabajos acerca de Europa y la ampliación hacia el Este o el Mediterráneo parecen arrojar resultados poco satisfactorios, pese a la permanencia de las hipótesis neoclásicas estándares y la lógica general.

\section{La visión del BID y el marco teórico del modelo}

Estevadeoral et al. (2000) lo indican claramente: "Nuestro análisis será muy puntual, porque ponemos en evidencia el elemento esencial del MERCOSUR: una disminución simultánea de los derechos de aduana internos y externos". ¿El MERCosuR representa un obstáculo o una etapa para el libre comercio multilateral? Según W. Ethier (1998), el nuevo regionalismo refleja el éxito de un sistema de comercio multilateral más que su fracaso. Utilizando un modelo de comercio con efectos de desvío ocasionados por los derechos de aduana de Krugman (1980), el análisis muestra que este tipo de regionalismo puede jugar

3 Véase también Piani y Kume (2000). 
un papel clave en la expansión y preservación del orden del comercio liberal. El fin de este modelo es "analizar los efectos en términos de bienestar de un arreglo comercial de tipo MERCOSUR sobre los miembros y los no miembros". Las funciones de utilidad $U$ en cada tipo de régimen comercial (preintegración, regionalismo cerrado y nuevo regionalismo) serán entonces comparadas entre los países miembros y aquellos que no pertenecen a la zona. Este análisis también tiene como objeto estudiar eventuales resultados y ventajas del lanzamiento del Área de Libre Comercio de las Américas (ALCA). Como lo sugirieron los autores del modelo del BID, dicho acuerdo podría constituir la experiencia más importante del nuevo regionalismo abierto en América, extendiéndose "desde Alaska hasta Tierra del Fuego (Estevadeordal et al., 2000:1-2). Destacan cuatro factores esenciales que influyen en los flujos comerciales:

- Fuerte disminución de las barreras al comercio entre los países miembros del MERCOSUR y el resto del mundo (véase Gráfica 1). Esos derechos de aduana alcanzaron una tasa de 14\% promedio en TEC, en el seno de la unión está sujeta a numerosas excepciones;

- La liberalización tuvo lugar antes del Tratado de Asunción de 1991;

- Asimetría (tamaño) en el seno del MERCosur mismo: la pareja Argentina-Brasil determina la mayor parte de los intercambios de exportación e importación; Paraguay y Uruguay contribuyen muy poco al volumen comercial total de la zona;

- Las tasas de cambio reales afectan el comercio intra y extramERCosur.

\section{El modelo del BID: método e hipótesis}

El interés por este modelo radica en la comparación de una función de utilidad en tres regímenes de comercio (análisis de estática comparativa).

- Los países 1 y 2 son aquellos que formaron un bloque comercial común en los regímenes B y C; de manera implícita, los autores del BID piensan en Brasil y Argentina;

- El país 3 es un país externo al bloque y considerado resto del mundo (RDM). Otra vez, de manera implícita, los diseñadores del BID piensan en el resto de América, es decir, el ALCA.

Estevadeoral et al. (2000) agregan dos hipótesis que simplifican el análisis formal y los cálculos:

1. No existen más que tres países en el mundo: los gustos de los consumidores, la tecnología y las dotaciones iniciales son idénticos (hipótesis de simetría entre los países);

2. El nivel de derechos de aduana resulta idéntico para todos los países y es del tipo todo o nada: los niveles de los derechos de aduana al comercio son iguales a $t$ (tarifa) o a 0 . El cambio del régimen A al B o al C es instantáneo.

\section{Desaarrollo}




\section{El modelo y los principales resultados}

Las principales ecuaciones conciernen a la función de utilidad del consumidor $U$, los precios de los bienes domésticos, los precios de los bienes extranjeros, el ingreso nacional, la cantidad de productos diferenciados potencialmente disponibles para $x$ consumidores, el salario en cada país y la demanda del factor trabajo. De esta forma, el efecto del MERCOSUR y de las reducciones de los derechos de aduana (según la cláusula de la nación más favorecida, NMF) se estudiarán en los países miembros de la zona (core) y los países extrazona (periphery).

La función de utilidad $U$ del régimen A (preintegración) será la base de comparación respecto de las funciones de utilidad de los regímenes B (regionalismo cerrado), y luego con relación al régimen $\mathrm{C}$ (nuevo regionalismo).

\section{Comparación del regionalismo cerrado (régimen $B$ )}

y la preintegración (régimen A)

- Régimen A (preintegración): situación original en la cual no existen bloques comerciales en el mundo. Los derechos de aduana $t$ se aplican a todo comercio entre 1, 2 y 3 .

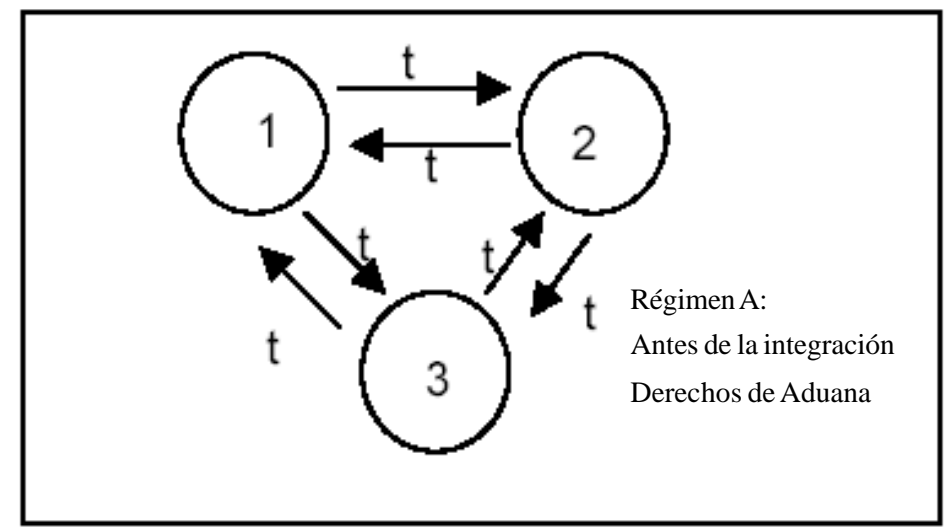

Gráfica 1.

- Régimen B (acuerdo de libre cambio de tipo regionalismo cerrado):4 los países 1 y 2 se encuentran integrados en una zona de libre comercio (sin derechos de aduana, DD). La nación 3 no forma parte del bloque. El comercio entre los países 1 y 3 así como entre los países 2 y 3 está sometido a derechos de aduana (DD: $t$ ).

4 Es el caso del antiguo regionalismo o de los acuerdos de libre comercio orientados hacia el mercado interno de la zona durante la industrialización, por sustitución de importaciones. 


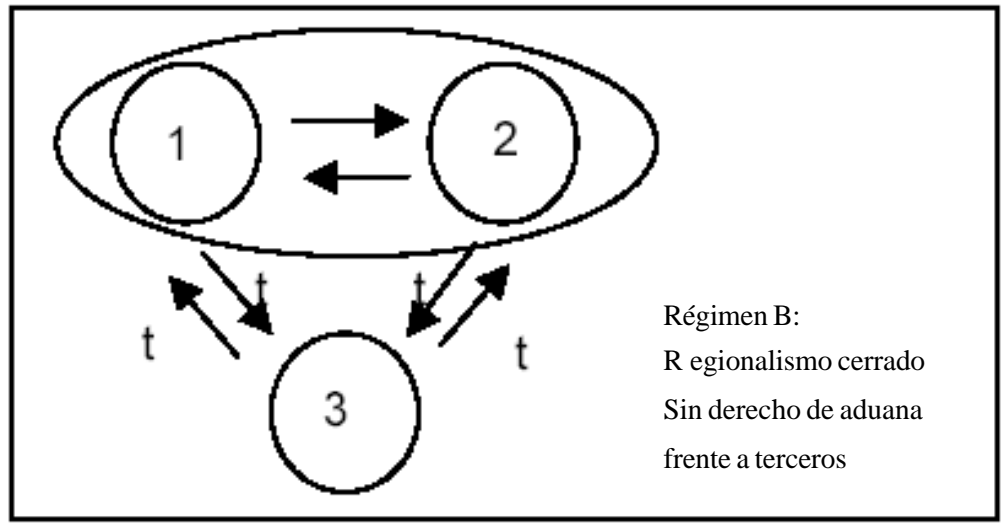

Gráfica 2.

Estos son, según los autores del modelo del BID, los efectos de una zona de libre comercio (zLC) tradicional (regionalismo cerrado): comparación de la función de utilidad de los países en los diferentes regímenes A y B:

- Resultado 1: si los países 1 y 2 forman una zona de libre intercambio (régimen B-RC), la intensidad del comercio entre los dos aumenta, mientras que el volumen de comercio entre las naciones integrantes de la zona y el país externo (país 3) disminuye. Es la expresión clásica del desvío comercial.

- Resultado 2: si las naciones 1 y 2 forman una zona de libre intercambio (RC), resultará que los términos del intercambio de los países miembros mejoran respecto de aquel de los territorios que se encuentran fuera de la zona.

- Resultado 3: si los países 1 y 2 forman una zona de libre intercambio (RC), el bienestar económico (función de utilidad) de los integrantes mejora, mientras que empeora el de los países que se encuentran fuera de la zona.

$U$ países miembro en régimen $\mathrm{B}(\mathrm{RC})>U$ países 1 y 2 en régimen A (preintegración) $\mathrm{y}$

$U$ países extrazona en régimen $\mathrm{B}<U$ países extrazona en régimen $\mathrm{A}$

Estos resultados no hacen más que comparar la situación previa a la integración (régimen A) con la situación del régimen B (regionalismo cerrado). Los resultados concernientes al nivel de comercio, los términos del intercambio y, sobre todo, la función de utilidad confirman el fundamento legal de reducir los derechos de aduana. Subrayan que el país ubicado fuera de la zona (país 3 o RDM) sufrirá con esta situación (función de utilidad que alcanza un nivel inferior al del régimen A). El establecimiento de un regionalismo cerrado 
entre Argentina y Brasil genera, por lo tanto, una reducción de la función de utilidad para el RDM y una disminución de los flujos de comercio con el RDM, en este caso, del ALCA.

La segunda fase de las comparaciones estudia las diferencias entre un nuevo regionalismo (régimen C) que simula el caso de un ALCA y una situación de preintegración (régimen A).

\section{Comparación del nuevo regionalismo y la preintegración}

- Régimen C: zona de libre intercambio de tipo MERCosur, incluyendo la cláusula de la nación más favorecida vis-à-vis del RDM.

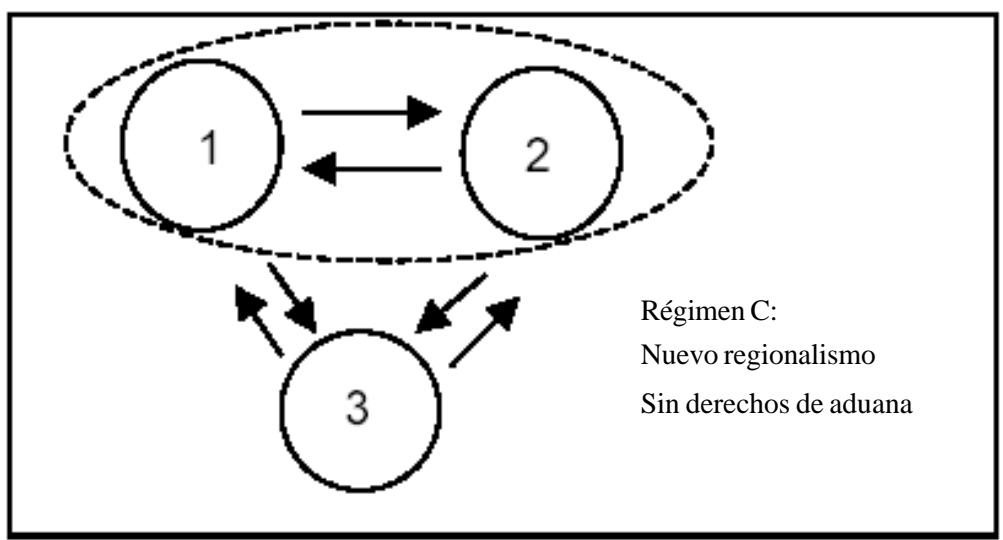

Gráfica 3.

Veamos ahora los resultados de una ZLC de tipo MERCosur (regionalismo abierto o nuevo regionalismo):

- Resultado 4: si los países 1 y 2 reducen a 0 los derechos de aduana entre ellos, aplican la cláusula de la nación más favorecida al país 3 (sin DD), y si éste también efectúa una reducción según esta misma cláusula (sin DD), entonces el comercio mundial aumentará.

- Resultado 5: si las naciones 1 y 2 reducen a 0 los derechos de aduana entre ellos, aplican la cláusula de la nación más favorecida al país 3 (sin DD), y si éste efectúa también una reducción según esta misma cláusula (sin DD), entonces el bienestar de todos los países se incrementará. En este caso, Argentina y Brasil (MERCosur) se benficiarían, pero también el ALCA.

$$
U \text { país } 1,2 \text { y } 3 \text { (régimen C) > } U \text { países } 1,2 \text { y } 3 \text { (régimen A) }
$$

La diferencia esencial concierne al régimen $\mathrm{C}$, el cual garantiza bienestar y un nivel de comercio superior al de la situación preintegración pero, sobre todo, beneficia a todos los países miembros de la unión aduanera (así como al RDM, es decir, al ALCA). 
¿Cuáles son las conclusiones principales de este modelo?

La ventaja del nuevo regionalismo sobre el regionalismo cerrado (así como la ausencia de integración con los DD altos). La integración de tipo nuevo regionalismo contribuye a la expansión del comercio mundial y preserva el libre comercio extrazona. En el régimen C (nuevo regionalismo), es posible alcanzar el bienestar de un país sin perjudicar el de los otros. Así, este análisis sugiere la probabilidad de que el nuevo regionalismo de tipo integración regional del MERCosur (con la reducción a 0 de los derechos de aduana hacia el RDM) contribuya no solamente a su propio bienestar económico, sino también al RDM o sea, el ALCA.

\section{Aportes y límites de este modelo de economía geográfica aplicado al MERCOSUR}

Presentamos en la sección anterior el modelo teórico del BID (hipótesis, resultados y conclusiones principales) que es claramente favorable al nuevo regionalismo y al ALCA. En la presente sección estudiaremos los aportes y límites de este tipo de modelo y su aplicación al caso del MERCOSUR por el BID.

Los aportes de este tipo de modelo aplicado al MERCOSUR

El objetivo de esta sección no es hacer una crítica teórica del enfoque de la economía geográfica ni de las nuevas teorías del comercio internacional, lo cual necesitaría nuevos estudios. Deseamos reflexionar acerca del fundamento legal de este tipo de enfoque aplicado al MERCOSUR y evaluar su pertinencia para una transformación productiva e igualitaria.

Esta experiencia en el MERCOSUR presenta un nuevo actor y encuentra en el nivel regional una nueva estrategia de desarrollo. Así, este modelo del BID demuestra que la integración no es causa del desvío en el comercio, como sugiere el modelo estándar Hos.

Los resultados de la comparación entre preintegración y regionalismo cerrado (régimen A y régimen B) confirman las ventajas de la integración regional y contribuyen a su reconocimiento como promotora del crecimiento y del bienestar suplementario.

Sin embargo, la comparación entre los regímenes A y C y los resultados que se obtengan nos llevarán a preguntarnos por las conclusiones de los autores de este modelo. En efecto, el regionalismo abierto (régimen $\mathrm{C}$ ) es preferible porque garantiza un nivel de bienestar superior a los miembros de la unión económica y a los no miembros, mientras que el régimen B (regionalismo cerrado) beneficia a los países integrantes en detrimento de los no integrantes (RDM). El regionalismo cerrado sería, entonces, una desviación del comercio y tendría como consecuencia una reducción del bienestar de las naciones extrazona. Por tal razón, es denunciado y criticado por los autores de dicho modelo.

No obstante, podemos lamentar la ausencia de comparación entre los regímenes B y C y entre éstos y el A (puramente hipotético), según los cuales, los países que forman una

\section{Desaarrollo}


zona económica se desconectan del mundo y se desarrollan autárquicamente vis-à-vis del RDM. Este punto nos lleva a los límites de dicha aplicación y de este tipo de modelo (que serán discutidos en la siguiente sección).

\section{Límites del modelo}

Más allá de las críticas externas en lo que a este modelo neoclásico se refiere (utilización de función de utilidad del consumidor, inconsistencia del régimen A en la historia, entre otros), vamos a subrayar ciertos límites intrínsecos. Señalaremos las críticas y las diferencias hechas entre la concepción de regionalismo abierto de la CEPAL en 1994 y el nuevo regionalismo de la economía geográfica y del BID.

Nuestras dos primeras críticas se refieren a las hipótesis simplificadoras del modelo (reducción instantánea de los derechos de aduana y países idénticos).

\section{Tres países idénticos}

Los autores del modelo del BID toman como hipótesis que las tres naciones son idénticas en todos los aspectos (preferencias de gusto, tecnologías, dotaciones iniciales). Esto resulta lógico si consideramos el fin de este tipo de patrones de economía geográfica. Este análisis busca explicar dónde están localizadas las actividades económicas. Las consideraciones iniciales no simétricas dificultan la identificación de las fuerzas endógenas que llevan a cierta localización de actividades. Si bien esta hipótesis simplifica mucho la presentación del modelo y la resolución formal final, es susceptible a muchas críticas.

Si los tres países son idénticos (y, sobre todo, si su tecnología, gustos y dotaciones iniciales lo son), intercambian en razón de la economías de escala y de la diferenciación de productos. Cabe preguntar si esto no signfica que las tres naciones forman en realidad un solo y único país. Esta observación implica discutir el concepto de país o región en este modelo y en el enfoque de la economía geográfica. Según ésta, "consideramos que un bloque comercial comprende un gran número de pequeñas unidades geográficas, provincias o regiones. Los países se componen de una o varias provincias o regiones y no juegan un rol explícito en el análisis" (Krugman, 1991b:320). Las entidades territoriales son puntos que revisten las especificidades establecidas por el modelo y el uso que le queremos dar (regiones, ciudades, países). Los término región y nación son ambiguos; definen una zona tan sólo sobre la base de "buenas razones únicamente económicas" y de las necesidades del analista. Sin embargo, en otro trabajo, el mismo autor señala que "las naciones tienen una importancia, dado que tienen gobiernos cuyas acciones políticas afectan a los movimientos de los bienes y de los factores de producción" (Krugman, 1991c:71-72). Así, esta pregunta fundamental no se responde y los modelos continúan utilizando indistintamente regiones, provincias o países. 
Por otra parte, las relaciones comerciales entre las naciones del MERCOSUR estuvieron determinadas largo tiempo por las economías del centro. ${ }^{5}$ Durante la industrialización, llevada a cabo por el sesgo de la ISI, y hasta fines de los años 1960, los dos grandes del MERCOSUR desarrollaron una relación comercial de espalda contra espalda. El ratio comercial era muy débil y el desarrollo recíproco representaba una situación en la cual los flujos comerciales se orientaban claramente hacia Estados Unidos, la Unión Soviética o Europa. ${ }^{6}$ Las fronteras entre Brasil y Argentina no se habían modificado lo suficiente para cambiar su relación comercial. Éstos son aspectos históricos, políticos y sociológicos que pueden explicar con mayor precisión esta configuración del comercio. En lo que respecta a los gustos simétricos entre los países representantes de la economía mundial del modelo, otros autores (como Furtado) ya explicaron e hicieron hincapié en los hábitos de consumo de los países subdesarrollados, donde las elites nacionales importan los bienes de las economías del centro dominante. ${ }^{7}$ La preferencia por una gran diversidad de productos ${ }^{8}$ —núcleo de la competencia imperfecta y de los modelos de geografía económica- no es un factor determinante en el proceso de integración económica entre naciones en vías de desarrollo, dentro del contexto de liberalización y de apertura. Sin embargo, vale la pena considerar las consecuencias en la estructura productiva.

Además, el modelo de Dixit-Stiglitz da cuenta de otros inconvenientes teóricos, como los señalados por Combes (2002):

- Existe un solo parámetro que sirve para la diferenciación de los productos, así como para los rendimientos crecientes;

- No existen interacciones estratégicas y, por lo tanto, tampoco el efecto de la competencia en las empresas. La estructura institucional y del mercado no tiene influencia en las estrategias de los agentes económicos. Esta hipótesis podría ser cierta en un contexto económico e institucional estable, pero es difícilmente aplicable a los países miembros del MERCOSUR, dados los cambios fundamentales que experimentaron por las reformas estructurales de fines de los años setenta;

5 Utilizaremos en este caso la terminología del análisis cepaliano y no el desvío de término que utiliza Krugman en su modelo centro periferia para distinguir las regiones integradas económicamente (core) de las que no lo estaban (periphery).

6 "En general, todos los factores y variables de comercio [países subdesarrollados en América Latina] se desarrollaron, en gran parte, en relación con las economías del centro. El comercio entre economías subdesarrolladas de la región latinoamericana fue entonces siempre insignificante o poco desarrollado" (Ffrench Davis, 1999:169).

7 Véase Celso Furtado (1976:53).

8 Véanse las ecuaciones (1), (5) y (5’) en el modelo del artículo en cuestión.

\section{Desaarrollo}


- La hipótesis de movilidad perfecta del factor trabajo puede ser pertinente para una pequeña extensión geográfica o en el seno de Estados Unidos, pero no lo será en esa medida para Europa o para una gran escala geográfica como el MERCOSUR. ${ }^{9}$

\section{Nivel de derechos de aduana instantánea (todo o nada)}

Ahora analizaremos la hipotesis (de Esteradeordal et al., 2001:183) de reducción a 0 de las barreras a los derechos de aduana entre los países miembros de un solo bloque comercial.

La hipótesis puramente simplificadora de la instantaneidad de la disminución de los derechos de aduana supone un contexto económico general que no provee explicaciones pertinentes acerca de los efectos de la integración económica; únicamente permite el análisis formal en un ámbito matemático. Esta hipótesis del nivel de derechos de aduana todo $o$ nada contradice las diferentes velocidades que disponen varios acuerdos de bloques comerciales de América. Según Delvin y Estevaeordal (2001), un sector fundamental, como la agricultura, registra, en el caso del MERCOSUR, los niveles más graduales de apertura. El análisis de la reducción gradual de los derechos de aduana al comercio podría ser útil para comprender la destrucción (y eventual reconstrucción) de los sectores industrial, agrícola y de servicios en las economías, así como sus efectos en la naturaleza y la intensidad del proceso dinámico de integración económica. ${ }^{10}$

Además, los niveles idénticos de derechos de aduana entre los países constituye otra hipótesis muy restrictiva y alejada de todo fundamento histórico. La comparación de los derechos de aduana en Estados Unidos, la Unión Europea, Asia y América Latina no permite sostener esta hipótesis, la cual, además, introduce la idea de un modelo homogéneo.

No existe un verdadero proceso de localización dinámico

o de aglomeración

El análisis estático comparativo no permite tomar en cuenta las transformaciones estructurales profundas que sufrieron la industria o el empleo durante este periodo. Sin embargo, dicho método de comparación entre una situación de autarquía y una de apertura resulta recurrente en el enfoque neoclásico. Aunque la orientación de la economía geográfica deba concentrarse en los efectos dinámicos de regionalización (en oposición a los efectos estáticos del desvío en el comercio), esta aplicación sólo analiza las ventajas de tipo ZLC para el MERCOSUR, mediante el análisis estático comparativo y la integración económica instantánea.

Véase, por ejemplo, Krugman y Venables (1995).

10 El MERCosur es una unión aduanera incompleta. Sin embargo, este modelo no trata claramente la diferencia entre uniones económica, aduanera completa o monetaria. 


\section{Ausencia de diferencias entre tipos de empresas}

Como ya mencionamos, este modelo ignora la influencia que tuvieron - en la configuración del comercio de los países del Cono Sur- sus relaciones comerciales con los países industrializados del centro. Uno de los vectores de esas relaciones fue la presencia de empresas transnacionales (ETN) en la zona. En ningún momento el análisis considera los distintos tipos de organización de las empresas y llama equivocadamente productor o empresa a realidades muy diferentes de las casas matrices, situadas en los países del centro: filiales, centros de I\&D, sucursales, centros de decisión, fábricas de producción o ensamblaje, entre otros. En realidad, la teoría microeconómica, tan rica para describir la firma, sus tomas de decisión y su organización, no se utiliza en este tipo de modelos. Se considera a la empresa a un bloque homogéneo y compacto, localizado en un sitio particular, sin tomar en cuenta sus diferentes dimensiones. Esta observación sobrepasa el caso de este patrón aplicado al MERCosur. En efecto, el modelo de Krugman no considera las decisiones de localización de la firma en función de factores cualitativos y organizacionales. ${ }^{11}$ En Brasil y en Argentina, las privatizaciones que tuvieron lugar en el momento de las reformas estructurales en todas las ramas de la industria y las finanzas (menos en la agricultura), llevaron a muchos economistas a hablar de transnacionalización de las economías nacionales. ${ }^{12}$ Resulta esencial distinguir entre fábrica de ensamblaje, centro de I\&D, filiales y casas matrices en el extranjero. Ignorar estos factores al estudiar las elecciones estratégicas de localización de las firmas (I\&D, ensamblaje, filiales, entre otras) torna parciales e insatisfactorios los resultados. En efecto, elementos como valor agregado, dinamismo de mercados y eventuales efectos de difusión del progreso técnico no son equivalentes entre los distintos tipos de empresas.

11 Los trabajos de Markusen tienden a remediar este límite. Chamboux Leroux (2000) ha dedicado varios estudios a corregir esta falta del modelo de base krugmaniana. De esta forma, observa que "el aporte del trabajo de Ekholm y Forsild (1997) al modelo de centro periferia de Krugman se funda en una redefinición de la firma y de su naturaleza. Uno de los límites evidentes del modelo de Krugman es la asimilación que se hace entre firma, empresa, fábrica, área de I\&D, casa matriz, filial, centro de decisión. Parece en efecto que la empresa constituye un bloque compacto que se desplaza al unísono. Esta 'reagrupación' resulta sin ninguna duda un elemento de aumento de las tendencias y en particular en el caso de la aglomeración de las empresas" (p. 601). Los trabajos de Ekholm, Forsild (1997) y la hipótesis del modelo con firmas horizontalmente integradas, aleja el pilar central del modelo centro periferia de Krugman, a saber, los rendimientos crecientes a escala dentro de la firma fábrica localizada. Por el contrario, los rendimientos a escala aparecen aun si una firma tiene fábricas localizadas en diferentes lugares. Así, no es necesario que una firma se localice en un solo sitio. Esto es posible en el modelo porque los costos fijos se encuentran asociados a la I\&D; por lo tanto, ésta puede difundirse libremente en el seno de la firma y entre sus diferentes lugares de producción (Chamboux Leroux, op. cit::602). Estos enfoques son más interesantes pero, en el caso de este modelo aplicado al MERCOSUR, no se les menciona.

12 Véanse Chusnovsky y López (2001); Basualdo (2000) para el caso argentino y Gonçalves (1999), para el brasileño.

\section{Desarrrollo}


Tal es, sin embargo, el caso de este modelo aplicado al MERCosur. En estos países, las elecciones de localización se hacen más por razones de competitividad, precio, relación de cambio o condiciones fiscales acordadas por los estados (o las provincias, por ejemplo, las cuestiones de la guerra fiscal en Brasil), que en función de las barreras aduaneras. ${ }^{13}$

\section{Falta de distinción sectorial e interés por la tecnología}

Lo único que importa son la apertura y el acceso a los capitales extranjeros, lo cual equivale, de hecho, a no tener política industrial sectorial. La competencia y la modernización benefician al conjunto de la economía. Las tecnologías (que juegan un papel esencial en las teorías del crecimiento endógeno) tienen consecuencias diferentes en países desarrollados y subdesarrollados. En razón de la configuración de la economía mundial y de su posición de dominación respectiva, así como de sus estructuras socioeconómicas diferentes, no podemos esperar las mismas consecuencias. Según Salama (1999), "las técnicas utilizadas, el grado de calificación y las formas de puesta en marcha son diferentes [entre países del centro y de la periferia], pero el grado de libertad en la elección de las técnicas y del tipo de calificación requerido no es infinito. Llevan la impronta de los segmentos producidos en el centro".

\section{Falta de consideración monetaria y financiera en un régimen} de acumulación de dominio financiero

Este último examen de la aplicación del modelo de economía geográfica al MERCosuR pretende recordar en qué nivel se sitúan los debates en torno a la integración regional en América Latina. Las explicaciones acerca de la localización de las actividades económicas que surgieron de los modelos tradicionales de economía geográfica pueden parecer menores frente al impacto del contexto macroeconómico. Elementos como volatilidad, inestabilidad, hiperinflación, megadevaluación, tipo de cambio y estrategias de las casas matrices en los países del centro dependen de la localización. ${ }^{14}$ Es cierto que los autores del modelo reconocen el papel central del tipo de cambio y de las variables monetarias, así como sus consecuencias en el comercio y en las evoluciones en la competitividad intrarregional. Incluso, precisan en ese artículo que:

13 Véase, por ejemplo, UNCTAD (2003:54), en el cual se cita el caso de Toyota, que anunció un proyecto de 200 millones de dólares en Argentina no en función de las barreras tarifarias o de la productividad, como lo suponen Darrigues y Montant (2001), sino a causa de la depreciación de la moneda de ese país y de la baja de costos que ello implica.

14 Algunos autores subrayaron la importancia de esas modificaciones haciendo referencia a un régimen de acumulación de dominio financiero, el cual tuvo su auge en el Cono Sur en los años noventa. Véanse Salama (1999) y Chesnais (1994 y 1996).

\section{Desaarrollo}


el comportamiento extra e intra-regional se halla influenciado por los tipos de cambio de los diferentes países entre ellos pero también vis-à-vis del RDM. Esta cuestión fue raramente tratada para el caso del MERCosur. Investigaciones futuras deberían combinar los efectos de las preferencias comerciales (integración) con aquellas de los cambios relativos de la competitividad relativa del comercio intra-regional. Esto cobra mucha importancia en el caso del MERCosur, porque los dos socios principales, Argentina y Brasil, conocieron episodios de hiperinflación, de fluctuaciones muy importantes de los tipos de cambio real y muchos intentos de estabilización durante el período 1985-1995" (Estevadeordal et al., 2000:15).

Pese a estos argumentos, los autores dejan tales consideraciones para trabajos futuros y continúan su análisis de la integración regional del Cono Sur sin atender estas variables. La dicotomía entre variables reales (comercio) y monetarias (tipo de cambio, moneda) no es nueva en el análisis neoclásico, para el cual la neutralidad monetaria es un postulado de base. No obstante, el estudio de los tipos de cambio y el análisis monetario distan de ser los padres pobres del análisis neoclásico. ${ }^{15}$

\section{Conclusión}

La importancia concedida en este artículo al modelo del BID (desarrollado por Estevadeordal et al., 2000) podría sorprender. Es, sin embargo, uno de los pocos documentos que aplica la economía geográfica al MERCosur. Sin detallar el génesis y la historia del trabajo de Estevadeordal et al. (2000), de su modelo pero, sobre todo, de sus resultados (para el regionalismo abierto y el ALCA) resulta interesante que, después de su aparición en forma de documento de trabajo (working paper), fue publicado en Journal of Economic Integration en junio 2001 en versión resumida. En efecto, la formalización (modelo, ecuación, esquemas de los tres regímenes) fue suprimida y reemplazada por una nota al pie de página (nota 2, p. 183) que remite al documento de trabajo:

En una versión previa (y más larga) de este artículo, presentamos un análisis formalizado del efecto del MERCOSUR sobre el bienestar gracias a un modelo simple de tres países. Como lo mostramos en ese documento, si el MERCOSUR reduce unilateralmente sus derechos de aduana vis-à-vis de los miembros de la zona como vis-à-vis de los otros países, se acerca a una situación de libre cambio en la cual todos los países (MERcosur y resto del mundo) salen ganadores. Este tipo de regionalismo abierto parece así más benéfico que la forma tradicional de integración regional (regionalismo cerrado) (Esteradeordal et al., 2001:183).

Los resultados del artículo resumido confirman las ventajas del regionalismo abierto para el conjunto de la economía mundial y apoyan el lanzamiento del ALCA. Encontramos

15 Citemos los trabajos pioneros de Ricardo, y los de Harrod (1939), Balassa (1964), Samuelson (1964), Mundell (1961), y los más recientes de Mc Kinnon, Dornbusch y Krugman (1995). Insistimos en que los estudiosos interesados en la influencia de los tipos de cambio en la integración regional aíslan los resultados que considera la economía industrial y a menudo optan por una solu ción tipo dolarización o régimen de currency board.

\section{Desarrrollo}


los resultados de dicho working paper durante la conferencia anual del BID, en marzo de 2002 ("Looking Beyond the Borders: Opportunities and Chanllenges of the New Regionalism") que produjo el último reporte de la institución en 2002: Beyond the Borders: The New Regionalism in Latin America. En este último texto, de amplia difusión, no se hace mención alguna (ni siquiera en la bibliografía) al documento de trabajo ni a su modelo imperfecto. Únicamente se hallan sus resultados positivos, que ya nadie menciona, pero que sirven de base a las discusiones y argumentaciones de más alto nivel acerca de las ventajas del regionalismo abierto vis-à-vis del regionalismo cerrado, de la apertura para todos los países, y de las posibilidades del ALCA de fomentar el bienestar de todos. Es por esta razón que damos mucha importancia a dicho trabajo y también a las necesarias críticas que amerita.

Los resultados de más de diez años de este tipo de regionalismo — las crisis económicas, políticas y sociales, las profundas mutaciones que han provocado en la estructura industrial, productiva y social (pobreza, desempleo, desigualdades) — ${ }^{16}$ nos inducen a ser más que prudentes respecto de las conclusiones optimistas del BID (pero también de la nueva CEPAL). Estos límites también se aplican a los enfoques en términos de economía geográfica al MERCOSUR. La no activación de la red de seguridad regional (desmercosurización), el incremento de la heterogeneidad productiva en Brasil y el empobrecimiento tecnológico en Argentina son manifestaciones que no invitan a abandonar la integración regional sudamericana, sino a orientarla hacia una reestructuración productiva regional que fomente la homogeneidad social.

\section{Bibliografía}

Basualdo, E., Concentración y centralización del capital en la Argentina durante la década del noventa, Quilmes, Universidad Nacional de Quilmes, 2000.

Baumont, C.; P.P. Combes, P.H. Derycke y H. Jayet, Economie géographique: Les théories à l'epreuve des faits, París, Economica, 2000.

BID, Economic and Social Progress in Latin America: 2002 report; Beyond Border, the New Regionalism in Latin America, Washington, 2002.

CEPAL, Statistical Yearbook for Latin America and the Caribbean, Santiago de Chile, CEPAL, 2003a.

- Panorama de la inserción internacional de América y el Caribe 2001-2002, Santiago de Chile, CEPAL, 2003b.

- El regionalismo abierto en América Latina y el Caribe, la integración económica al servicio de

16 Véase Saludjian (2004b y 2005). la transformación productiva con equidad, Libros de la CePal núm. 39, Santiago de Chile, 1994.

Chamboux-Leroux, J.Y., Approches théoriques des disparités régionales: application au cas Mexicain, tesis de doctorado en ciencias económicas, París, Universidad de París, 2000.

Chesnais, F. (coordinador), La globalisation financière: Génèse, coûts et enjeux, París, La Découverte-Syros, 1996.

- Les pièges de la finance mondiale, París, La Découverte-Syros, 2000.

Chudnovsky, A. y A. López, La transnacionalización de la economía argentina, Buenos Aires, EUDEBACENIT, 2001.

Combes, P.P., Economic Geography Theory, Seminario a la segunda escuela de primavera de economía geográfica, París, Universidad de Pau, 2002.

\section{Dêsaarrollo}


Darrigues, F. y J.M. Montaud, "Intégration économique et agglomération des activités industrielles dans le MERCOSUR", Working paper presenté au colloque "Intégration Régionale et Développement" held in Aix-en-Provence, France, 2001

Delvin R. y A. Estevadeordal, "What's New in the Regionalism in the Americas?", Working Paper 6, INTAL-ITD-STA, 2001, www.iadb.org/intal

Estevadeordal, A.; J. Goto y R. Saez, "The New Regionalism in the Americas: the case of MERCOSUR", en Journal of Economic Integration, vol. 16, núm. 2, 2001.

, The New Regionalism in the Americas: the case of MERCOSUR, Working Paper, INTAL-STA-ITD, 2000, $52 \mathrm{pp}$.

Ethier, W., "The New Regionalism", en Economic Journal, núm. 108, 1998.

Ffrench-Davis, R., Macroeconomía, comercio y finanzas para reformar las reformas en América Latina, Santiago, CEPAL-Mc Graw Hill, 1999.

Furtado, C., Le mythe du développement économique, París, Anthropos, 1976.

Gonçalves, R., Globalização et desnacionalização, São Paulo, Paz e Terra, 1999.

INTAL, Informe MERCOSUR núm. 8, periodo 2001-2002, 2003, www.iadb.org/intal

Katz, J., Structural Reforms, Productivity and Technological Change in Latin America, CEPAL Book, núm. 64, Santiago de Chile, 2001.

Krugman, P., Development, Geography and Economic Theory, мIт Press, 1995.

-, Currencies and Crises, MIT Press, 1995.

, "Increasing Returns and Economic Geography", en Journal of Political Economy, vol. 99, núm. 3, 1991a.

-, The Move Toward Free Trade Zones, Federal Reserve Bank of Kansas City Economic Review, noviembre-diciembre, $1991 \mathrm{~b}$.

-, Geography and Trade, MIT Press, 1991c.

Krugman P. y A. Venables, "Globalisation and the inequality of nations", en Quarterly Journal of Economics, núm. 110, 1995.

Kuwayama, M., "Open Regionalism in Asia Pacific and Latin America: a survey of the literature",
Serie Commercio Internacional de la CEPAL, núm. 4, Santiago de Chile, 1999.

Piani G. y H. Kume, "Fluxos bilaterais de comércio e blocos regionais: uma aplicação do modelo gravitacional”, texto para discusión núm. 749, IPEA, julio de 2000.

Salama, P., "Globalisation, inégalités territoriales et salariales", miméo Greitd-CEDI, Universidad de París 13, 1999.

Saludjian, A., "De la Volatilité des Salaires à la Croissance Excluante dans le MERCOSUR 19912003: une Etude Statistique", en Cuadernos PROLAM/USP, vol. 02, año 3, Universidade de São Paulo, 2005, pp. 29-46, disponible en Internet, www.usp.br/prolam/downloads/2004_2_2.pdf.

, "Trajectoires de croissance, régime de change et volatilité dans le MERCOSUR: Quelques éléments d'analyse empirique", en Problemas del Desarrollo, Revista Latinoamericana de Economía, vol. 35, núm. 139, México, unAMIIEC, 2004a, disponible en Internet: http:// www.ejournal.unam.mx/problemas_des/pde 139/PDE13904.pdf.

—_ Hacia otra integración sudamericana: críticas al MERCOSUR liberal, Buenos Aires, Libros del Zorzal, traducción de la tesis de doctorado en ciencias económicas en la Universidad de París $13,2004 \mathrm{~b}$.

Siroën, J.M., "La théorie de l'échange international en concurrence monopolistique, une comparaison des modèles", en Revue Economique, vol. 39, núm. 3, mayo de 1988, pp. 511-544.

Stiglitz J. y A. Dixit, "Monopolistic Competition and Optimum Product Diversity", in Amercian Economic Review, núm. 67, 1977.

Terra M.I. y A. Gigliotti, "MERCosUR: localización de la producción, un modelo de geografía económica", en Revista de Economía del Banco Central de Uruguay, vol. 2, núm. 1, segunda época, mayo de 1995.

UNCTAD, World Investment Report 2003, FDI policies for development: national and international perspectives, Ginebra, 2003.

Viner, J., The Custum Union Issue, Nueva York, Carnagie Endowment for International Peace, 1950. 